\title{
Comment on "Projective Quantum Monte Carlo Method for the Anderson Impurity Model and its Application to Dynamical Mean Field Theory"
}

\author{
M. I. Katsnelson ${ }^{1, *}$ \\ ${ }^{1}$ Institute for Molecules and Materials, Radboud University Nijmegen, 6525 ED Nijmegen, The Netherlands \\ PACS numbers: 71.10.-w, 02.70.Ss, 71.27.+a
}

In a recent Letter 1], Feldbacher, Held, and Assaad have proposed a new scheme for the Quantum Monte Carlo simulations of the impurity Anderson model. This method is supposed to calculate the average of an operator $\mathcal{O}$ over the ground state of the Hamiltonian $H$ via the expression

$$
\langle\mathcal{O}\rangle_{0}=\lim _{\theta \rightarrow \infty} \lim _{\beta \rightarrow \infty} \frac{\operatorname{Tr}\left[e^{-\beta H_{T}} e^{-\theta H / 2} \mathcal{O} e^{-\theta H / 2}\right]}{\operatorname{Tr}\left[e^{-\beta H_{T}} e^{-\theta H}\right]}
$$

where $H_{T}$ is an appropriate trial Hamiltonian (see Ref 1 , Eq.(3)). The main requirement to the choice of $H_{T}$ is that its ground state can not be orthogonal to the ground state of the Hamiltonian under consideration $H$. In real calculations, the authors of Ref 1 have taken $H$ as the impurity Anderson Hamiltonian and $H_{T}$ as its noninteracting part, i.e. the Anderson Hamiltonian without the Hubbard interaction term $-U / 2\left(n_{\uparrow}-n_{\downarrow}\right)^{2}$ where $n_{\sigma}$ is the operator of number of localized electrons with spin projection $\sigma=\uparrow, \downarrow$ on the impurity site. Unfortunately, this choice is not consistent with the basic restriction mentioned above due to the Anderson orthogonality catastrophe [2, 3]. Anderson has proven that in thermodynamic limit $N \rightarrow \infty$ (where $N$ is the number of electrons) a local perturbation leads to complete reconstruction of the ground state of fermionic system in such a way that the overlap of the "old" and "new" groundstate way functions, $\left|\Psi_{T}\right\rangle$ and $|\Psi\rangle$, is proportional to $N^{-\alpha}$ where for the case of spherically symmetric perturbation potential

$$
\alpha=\sum_{l=0}^{\infty}(2 l+1)\left(\frac{\delta_{l}}{\pi}\right)^{2},
$$

$\delta_{l}$ being the scattering phases at the Fermi level with orbital quantum number $l$. Later this expression was generalized to the case of arbitrary local perturbation [4]:

$$
\alpha=\frac{1}{2}\left(\frac{1}{2 \pi i}\right)^{2} \operatorname{Tr}(\ln \widehat{S})^{2}
$$

where $\widehat{S}$ is the scattering matrix of the local perturbation at the Fermi surface. Original derivation of the Anderson orthogonality catastrophe was done for the case of local one-body potential; however, later it has been proven also for the Hubbard interaction term [5]. Taking into account the Friedel sum rule for the Anderson model 6, 7] one can demonstrate that $\alpha$ is proportional to $\left(n_{d}-n_{d}^{0}\right)^{2}$ where $n_{d}, n_{d}^{0}$ are the impurity occupation numbers for the Hamiltonians $H$ and $H_{T}$, correspondingly. For a special case of symmetric half-filled Anderson model one has $\alpha=0[\underline{8}]$; however, to satisfy this in a generic case one has to know already an exact answer for $n_{d}$ which makes the whole scheme unpractical, at least, in the context of the dynamical mean-field theory. For the case of non-degenerate Anderson model the value $n_{d}$ can be calculated exactly by the Bethe Ansatz method [9].

Thus, for the infinite system the ground state does not contribute to both numerator and denominator of the ratio (11) and, instead of being the average over the ground state the latter is rather some weighted average over excited states.

The "orthogonality catastrophe" is a generic and fundamental property of many-body fermionic systems (see, e.g., discussion in Ref 10 ) and should be carefully taken into account at the development of different projection techniques.

* Electronic address: m.katsnelson@science.ru.nl

[1] M. Feldbacher, K. Held, and F. F. Assaad, Phys. Rev. Lett. 93, 136405 (2004).

[2] P. W. Anderson, Phys. Rev. Lett. 18, 1049 (1967); Phys. Rev. 164, 352 (1967).

[3] S. V. Vonsovsky and M. I. Katsnelson, Quantum Solid State Physics (Springer, Berlin etc., 1989), Sect. 5.7.

[4] K. Yamada and K. Yosida, Prog. Theor. Phys. 62, 363 (1979).

[5] K. Yamada and K. Yosida, Prog. Theor. Phys. 59, 1061 (1978); ibid. 60, 353 (1978).

[6] D. C. Langreth, Phys. Rev. 150, 516 (1966).

[7] A. C. Hewson, The Kondo Problem to Heavy Fermions (Cambridge University Press, Cambridge, 1993), Sect. 5.2 .

[8] The absence of the orthogonality catastrophe for this special case was proven in this way by M. Feldbacher, K. Held, and F. F. Assaad, Phys. Rev. Lett. 96, 139702 (2006).

[9] P. B. Wiegmann and A. M. Tsvelick, J. Phys. C 16, 2281 (1983).

[10] P. W. Anderson, The Theory of Superconductivity in the High- $T_{c}$ Cuprates (Princeton University Press, Princeton, 1997). 